\title{
Ermittlung der wesentlichen Einflussfaktoren zur Optimierung der Entzugsleistung von Erdwärmetiefsonden
}

Zusammenfassung: Eine möglichst gute Kenntnis der geologischen und hydrogeologischen Verhältnisse des Untergrundes, sowie der anlagenspezifischen Einflussfaktoren ist Grundvoraussetzung für eine ökonomische und ökologische Anlagendimensionierung und einen ebensolchen Anlagenbetrieb. Die Eigenschaften des Untergrundes bestimmen dabei die Wahl einer bestimmten Anlagenvariante, wie zum Beispiel Grundwasserwärmepumpe oder Erdwärmesonde, sowie die spezifische Auslegung der gewählten Variante. Die dabei wesentlichen Parameter sind die Wärmeleitfähigkeit $\lambda$ und die Wärmekapazität c. Vergleichsmessungen dieser Parameter in Situ ergeben dabei im Vergleich zu einschlägigen Literaturwerten ähnliche Ergebnisse. Bei den Festgesteinen lassen sich Abweichungen dieser Parameter auf Schwächungen (Klüfte, Mikrorisse) und Verwitterung der untersuchten Gesteine zurückführen. Es ist daher durchaus davon auszugehen, dass im massigen ungestörten Gesteinsverband höhere Wärmeleitfähigkeiten als in oberflächennahen Aufschlüssen gemessenen auftreten.

Bei den Lockergesteinen sind die Körnungslinie sowie Lagerungsdichte und Wassergehalt maßgebend für die Wärmeleitfähigkeit. Unterschiede zu den Literaturangaben zeigen sich besonders bei Schottern. Diese weisen im bergfeuchten Zustand höhere $\lambda$-Werte auf als in der Literatur angegeben.

Weiters konnte gezeigt werden, dass mit Hilfe der ortsauflösenden faseroptischen Temperaturmessung der Einfluss der Geologie und Hydrogeologie auf die sich im Betrieb der Erdwärmesonde einstellende Soletemperatur dargestellt werden kann. Ein homogener geologischer Untergrundaufbau spiegelt sich in einem stetig ansteigenden Temperaturprofil der Sole über die gesamte Sondenlänge wieder. Bei einem inhomogenen Untergrundaufbau zeigt auch der Verlauf der Soletemperatur Unstetigkeiten. Eine Differenzierung in unterschiedliche Abschnitte ist möglich.

Bei Erdwärmetiefsonden ist die Art und Güte der verwendeten Bohrlochver- pressung ebenfalls ein wichtiger Einflussfaktor für die optimale Nutzung der Wärmegewinnungsanlage. Aus der Untersuchung von sieben am Markt angebotenen Fertigprodukten sowie von drei Rezepturen (Baustellenmischungen) zur Herstellung von Verpressmaterialien für Erdwärmesonden können in Hinblick auf die thermische Eigenschaften und die wasserwirtschaftlich relevanten Aspekte eine nachhaltige und dauerhafte hydraulische Abdichtung sowie eine optimale Wärmeleitfähigkeit bei geringem thermischen Bohrlochwiderstand als maßgebenden Anforderungen festgehalten werden.

Auf die Gewährleistung einer nachhaltigen und dauerhaften hydraulischen Abdichtung haben bereits der Anmischvorgang sowie die damit einhergehenden Suspensionsparameter großen Einfluss. Besondere Bedeutung kommt dabei den Parametern Fließgrenze, Filtratwasserabgabe und Absetzmaß zu. Wie in den Untersuchungen gezeigt werden konnte, beeinflusst die Gefügeschädigung aufgrund der Frost-Tauwechselbeanspruchung die Dichtigkeit als auch die Wärmeleitfähigkeit des Verpressmaterials. Durch Mikrorisse und Ablösungen in den Übergangsbereichen wird der Wärmefluss gestört und die Wärmestromdichte sinkt. Generell sollte thermisch verbessertes Verfüllmaterial verwendet werden. Auch ist bei der Anlagendimensionierung darauf zu achten, dass Soletemperaturen unter Null Grad nicht über einen langen Zeitraum auftreten.

Determining the main factors for optimising the extraction capacity of geothermal heat exchanger

Summary: Comprehensive knowledge of the geological and hydrogeological conditions of the ground and the special features of the geothermal system used is vital for the economical and ecological design and operation of such installations. The properties of the ground will decide the selection of a specific plant alternative, such as groundwater heat pump vs. geothermal heat exchanger, and the rating of the selected alternative. The parameters to be considered are heat conductivity $\lambda$ and heat capacity. Comparative in situ measurement of these parameters yields similar results to the values found in the relevant literature. Deviant results in rock suggest the presence of discontinuities, such as joints and micro cracks, as well as weathering. The assumption is therefore that massive undisturbed rock yields higher heat conductivity results than near-surface zones.

The heat conductivity of loose soil is a function of grading curve as well as density and moisture content. Differences from the values given in the relevant literature are mainly found in moistly gravels, which yield higher $\lambda$ values.

With the help of fiber optic temperature measurement the geological and hydrogeological influence on the brine temperature of a bohrehole heat exchanger can be shown. A homogeneous geological ground structure is reflected by a steadily increasing brine temperature profile over the length of the borehole, while unsteady temperature development suggests ground discontinuities. It is thus possible to identify different sections.

Another important factor for optimal heat extraction from borehole heat exchangers is the type and quality of the used grout. Testing seven commercial products and three mix formulas (on-site mixes) for grouting have led to the conclusion that sustainable and permanent hydraulic sealing of boreholes as well as optimal heat conductivity along with a low thermal borehole resistivity are essential requirements.

An important factor for ensuring sustainable and permanent hydraulic sealing is already the mixing process and the suspension parameters involved, in particular liquid limit, filtrate bleeding and slump. As shown in the tests, structural damage from freezing and thawing tends to affect grout impermeability and heat conductivity. Micro cracks and spalling in transition areas disturb and reduce the heat flux through the material. So it is important to 


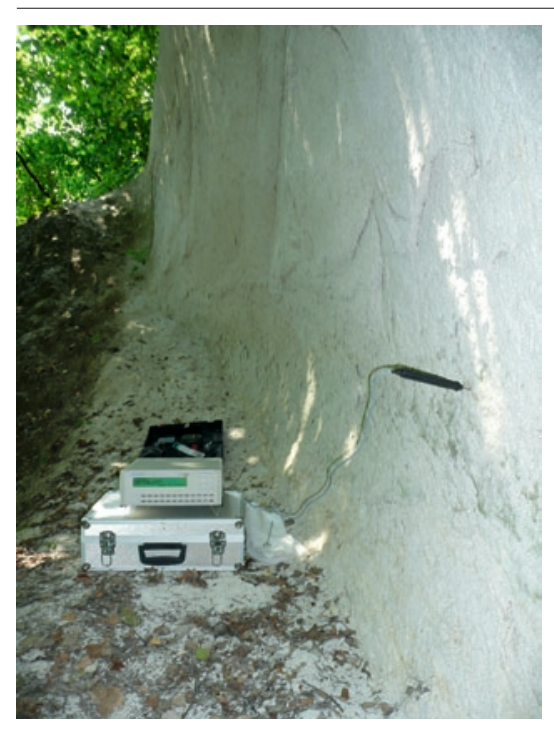

Abb. 1: Messungen im Gelände.

use thermally improved backfill material, and to pay attention on a brine temperature above zero degree in designing geothermal systems.

\section{Geologische und hydrogeo- logische Einflussfaktoren}

Die Wärmeentnahme durch Erdwärmesonden (Erdwärmesonde als Wärmesenke) verursacht im Untergrund, je nach Entzugsleistung und -dauer die Ausbildung eines mehr oder weniger ausgeprägten thermischen Entzugstrichters. Dies ist vergleichbar mit dem Absenktrichter um einen Förderbrunnen in der Grundwasserhydraulik.

Zwei thermische Größen sind dabei von wesentlicher Bedeutung:

\section{- Wärmekapazität C (J / kg K)}

Oberhalb des absoluten Nullpunktes hat der Boden einen Wärmeinhalt, der in Joule pro Gramm $(\mathrm{J} / \mathrm{g})$ oder in Joule pro $\mathrm{cm}^{3}\left(\mathrm{~J} / \mathrm{cm}^{3}\right)$ angegeben werden kann. Da diese Größe schwer zu erfassen ist, wird häufig die Wärmemenge angegeben, die in einer definierten Bodenmenge eine bestimmte Temperaturänderung hervorruft. Die entsprechende physikalische Größe nennt man spezifische Wärmekapazität $\mathrm{Cp}(\mathrm{J} /$ $\mathrm{kg} \mathrm{K}$ ). In der Literatur wird häufig die Wärmekapazität pro Volumeneinheit $\mathrm{Cv}\left(\mathrm{J} / \mathrm{m}^{3} \mathrm{~K}\right)$, die so genannte volumetrische Wärmekapazität verwendet (Hartge und Horn 1999). Sie kann über die Kenntnis der Lagerungsdichte aus der spezifischen Wärmekapazität berechnet werden $\left(\mathrm{Cv}=\mathrm{Cp}{ }^{*} \mathrm{D}\right)$.

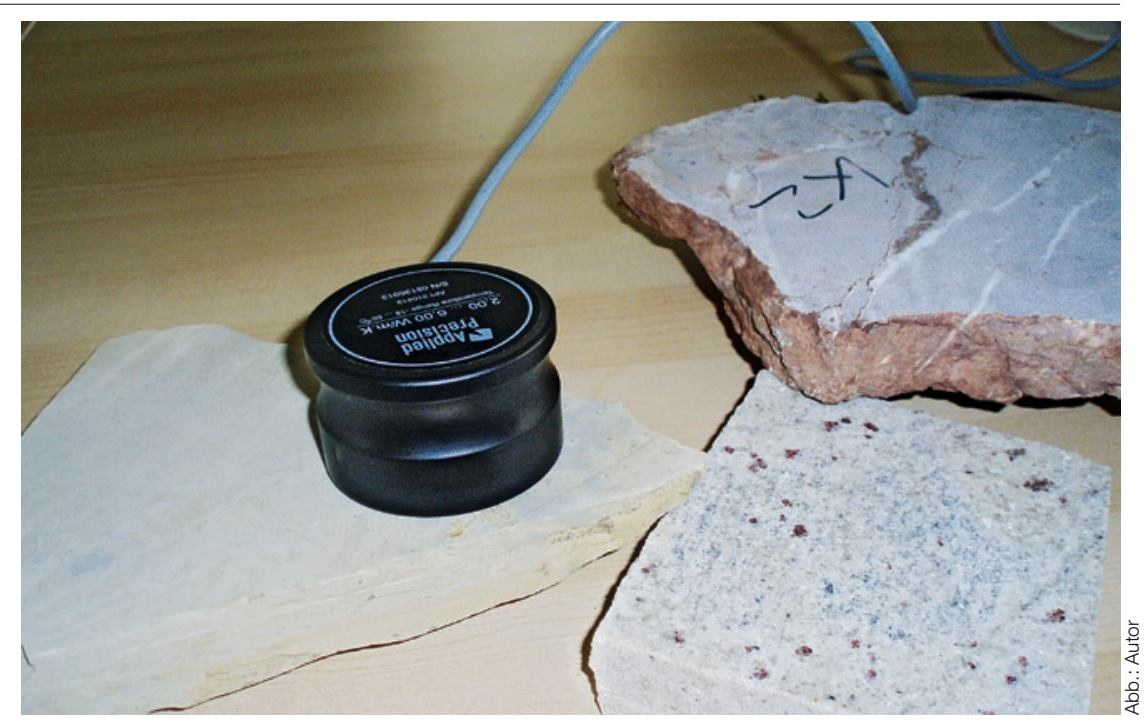

Abb. 2: Messungen an Handstücken.

- Thermische Leitfähigkeit -Wärmeleitfähigkeit $\lambda(\mathrm{W} / \mathrm{m} \mathrm{K})$

Die Wärmeleitfähigkeit $\lambda$. ist das Vermögen einer Substanz (Festkörper, Flüssigkeit oder Gas), thermische Energie in Form von Wärme zu transportieren. Sie gibt an, welche Energiemenge pro Sekunde durch einen Körper, der die Querschnittsfläche von $1 \mathrm{~m}^{2}$ und die Länge von $1 \mathrm{~m}$ hat fließt, wenn die Temperaturdifferenz zwischen beiden Seiten $1 \mathrm{~K}$ beträgt.

1.1. Direkte (quantitative) Bestimmung der thermischen Eigenschaften des Untergrundes - Messung der Wärmeleitfähigkeit und der Wärmespeicherkapazität

Im Zuge der Studie „Bestimmung der Wärmeleitfähigkeit verschiedener Gesteine Oberösterreichs", beauftragt von der Abteilung Wasserwirtschaft, Grund und Trinkwasserwirtschaft des Landes Oberösterreich, erfolgten Messungen der Wärmeleitfähigkeit und der Wärmekapazität an oberflächennah vorkommenden Locker- und Festgesteinsformationen im Bundesland Oberösterreich. Zusätzlich dazu wurden die generellen geologischen und geotechnischen Parameter bestimmt. $\mathrm{Zu}$ diesen zählen bei Lockergesteinen die Korngrößenverteilung, der Wassergehalt und die ungestörte Dichte, bei Festgesteinen die Rohdichte. Der Untersuchungsumfang der Studie umfasste 80 Messstellen, 50 davon in Lockergesteinen und 30 in Festgesteinen.

Die Auswahl und Dokumentation repräsentativer Aufschlüsse sowie die Durchführung von geotechnischen Feld- und Laborversuchen erfolgte gemeinsam mit der Oberösterreichische Boden- und Baustoffprüfstelle GmbH.

Die Bestimmung der thermischen Parameter erfolgte dabei mit dem mobilen Gerät ISOMET 2104 der Firma Applied Precision. Die Messung basiert auf der Analyse der Temperaturantwort des untersuchten Materials auf einen Wärmeimpuls. Dieser wird durch ein Widerstandsheizelement, welches in der Messsonde integriert ist, erzeugt. Aus den gemessenen Temperaturverläufen während der Aufheiz- und Abkühlphase wird die Wärmeleitfähigkeit $\lambda$ und die volumetrische Wärmekapazität cv berechnet. Als Messsonde stehen - je nach Materialbeschaffenheit - Einstichsonden als auch Auflegesonden zur Verfügung. In den beiden Abbildungen 1 und 2 sind Messungen mit Einstichsonden an Aufschlusspunkten im Gelände und Auflegesonden an Handstücken dargestellt.

Der Wassergehalt der Lockergesteine kann dabei durchwegs mit bergfeucht beschrieben werden. Die in der Literatur beschriebenen Extreme, trocken bzw. wassergesättigt, wurden bei keiner Messstelle angetroffen. Für ausgewählte Messstellen erfolgte ebenfalls die Ermittlung der in situ Dichte, bzw. der Rohdichte. Bei Lockergesteinen wurde zusätzlich die Sieblinie und der Wassergehalt festgestellt.

In der einschlägigen Fachliteratur wird meist auf die VDI 4640, Blatt 1, Tabelle 1 verwiesen. Darin sind Rechenwerte für die Wärmeleitfähigkeit und die volumetrische Wärmekapazität, geordnet nach Gesteinsarten, angegeben. In $\mathrm{Ta}$ belle 1 sind gemessene Werte der Wärmeleitfähigkeit und Wärmekapazität diesen 


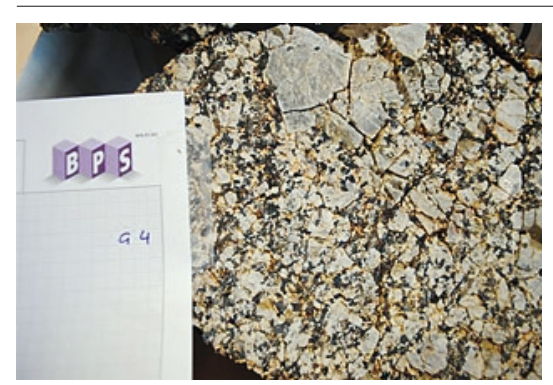

Abb. 3: frische Schnittfläche Weinsberger Granit.

Literaturwerten (Auszug VDI 4640, Blatt 1, Tabelle 1) gegenübergestellt.

Die gemessenen Werte der kristallinen Festgesteine liegen durchwegs am unteren Ende der in der Literatur angegebenen Bandbreite der Wärmeleitfähigkeiten. Deutlich ist dies bei den gemessenen Gneis- und Granitproben ersichtlich. Der geringste Wert von $\lambda=1,56 \mathrm{~W} / \mathrm{mK}$ wurde an einer Probe Freistädter Granodiorits gemessen und der höchste Wert von $\lambda=7,04 \mathrm{~W} / \mathrm{mK}$ zeigte sich an einer Probe eines quarzitischen Ganggesteins. Die starken Unterschiede sind einerseits auf den Mineralbestand und andererseits auf den Verwitterungszustand der Gesteine zurückzuführen. In Abbildung 3 ist exemplarisch dafür die Schnittfläche der Granitprobe G4 (Weinsberger Granit) gezeigt. Darauf sind deutlich die Klüftigkeit sowie die rostbraunen Flecken aufgrund von Verwitterung erkennbar. Da die Probe- nahme in oberflächennahen Aufschlüssen erfolgten, ist generell nicht mit einem massigen ungestörten Gesteinsverband zu rechnen.

Es ist daher davon auszugehen, dass die beprobten Gesteine aufgrund von Schwächungen (Klüfte, Mikrorisse) und Verwitterung geringere Wärmeleitfähigkeiten zeigen als sie im massigen ungestörten Gesteinsverband vorzufinden wären.

Durchwegs mit den Literaturangaben vergleichbare Werte zeigten die gemessenen sedimentären Festgesteine, hier speziell die Kalke und Dolomite. Die gemessenen Proben zeigten im Gegensatz zu den Kristallinproben keine erkennbare Klüftigkeit und Verwitterung. Besonders die Dolomite stellten sich als sehr homogen und feinkörnig dar, was sich in deren relativ hohen Wärmeleitfähigkeiten widerspiegelt.

Die gemessenen Werte erscheinen hier durchaus repräsentativ für den ungestörten massigen Gesteinsverband.

Die gemessenen Schlierformationen stellen sich mit Ausnahme des Ottnanger Schliers als relativ einheitlich dar. Die gemessenen Wärmeleitfähigkeiten liegen zwischen $\lambda=1,19$ und $1,75 \mathrm{~W} / \mathrm{mK}$. Unterschiede zwischen den einzelnen Varietäten ergeben sich aufgrund unterschiedlicher Kornzusammensetzung, Dichte und Homogenität. Weiters erodiert der Schlier an der Geländeoberfläche sehr stark, wo- durch sich Trennflächen entlang der sedimentären Schichtung bilden.

Wie bei den kristallinen Festgesteinen ist auch bei den Schlierformationen durchaus davon auszugehen, dass die oberflächennah gemessenen Gesteinsformationen aufgrund von Schwächungen und Verwitterung geringere Wärmeleitfähigkeiten zeigen als sie im massigen ungestörten Gesteinsverband auftreten. Die gemessenen Wärmekapazitäten liegen durchwegs im Bereich der Literaturangaben.

Eine große Variabilität zeigen die gemessenen Wärmeleitfähigkeiten der Lockergesteine.

Die gemessenen Sande weisen eine Bandbreite von $\lambda=0,33$ bis $2,46 \mathrm{~W} / \mathrm{mK}$ auf. Der geringe Wert von $\lambda=0,33 \mathrm{~W} / \mathrm{mK}$ wurde in einem Aufschluss der Enzenkirchner Sande gemessen. Es handelt sich dabei um stark durchwühlte, aufgelockerte Sande. Dies zeigt auch die relativ niedere Dichte von $\rho=1,62 \mathrm{~g} / \mathrm{cm}^{3}$. Der maximal gemessene Wert von $\lambda=2,46 \mathrm{~W} / \mathrm{mK}$ ergab sich an einem Aufschluss der Pleschinger Sande. Hier wurde auch die höchste in situ Dichte bei Sanden gemessen $\left(\rho=2,0 \mathrm{~g} / \mathrm{cm}^{3}\right)$.

Hohe in situ Dichten mit entsprechend geringen Luftporenanteilen bedingen hier hohe Wärmeleitfähigkeiten. Im Vergleich zu den Literaturangaben stimmen die Messwerte der Wärmeleitfähigkeit gut mit den angegebenen Rechenwerten überein.

TABELLE 1

Zusammenstellung von gemessenen Werten und Literaturwerten für $\lambda$ und $c_{v}$

\begin{tabular}{|c|c|c|c|c|c|c|c|}
\hline \multirow{2}{*}{ Gestein } & \multirow{2}{*}{$\begin{array}{l}\text { Dichte } \\
\left(\mathrm{g} / \mathrm{cm}^{3}\right)\end{array}$} & \multicolumn{2}{|c|}{ Wärmeleitfähigkeit $\lambda$ (W/mK) } & \multirow{2}{*}{$\begin{array}{l}\text { vol. Wärme- } \\
\text { kapazität } \\
\left(\mathbf{M J}^{3} \mathbf{m} \text { k) }\right.\end{array}$} & \multirow{2}{*}{$\begin{array}{l}\text { Dichte, } \\
\text { gemessen } \\
\left(\mathrm{g} / \mathrm{cm}^{3}\right)\end{array}$} & \multirow{2}{*}{$\begin{array}{l}\text { Wärmeleitf. } \\
\text { gemessen } \\
\text { (W/mK) }\end{array}$} & \multirow{2}{*}{$\begin{array}{l}\text { vol. Wärmekap. } \\
\text { gemessen } \\
\left(\mathrm{MJ} / \mathrm{m}^{3} \mathrm{k}\right)\end{array}$} \\
\hline & & von-bis & Rechenwert & & & & \\
\hline \multicolumn{8}{|l|}{ Festgesteine } \\
\hline Granit & $2,4-3,0$ & $2,1-4,1$ & 3,4 & $2,1-3,0$ & $2,53-2,67$ & $1,56-7,04$ & $1,55-2,19$ \\
\hline Gneis & $2,4-2,7$ & $1,9-4,0$ & 2,9 & $1,8-2,4$ & $2,59-2,66$ & $1,83-2,50$ & $1,60-1,88$ \\
\hline Kalkstein & $2,6-2,7$ & $2,5-4,0$ & 2,8 & $2,1-2,4$ & $2,66-2,73$ & $2,16-2,87$ & $1,82-2,09$ \\
\hline Sandstein & $2,2-2,7$ & $1,3-5,1$ & 2,3 & $1,6-2,8$ & $2,19-2,54$ & $1,43-3,74$ & $1,47-2,02$ \\
\hline Ton/Schluffstein & $2,5-2,6$ & $1,1-3,5$ & 2,2 & $2,1-2,4$ & $2,02-2,15$ & $1,28-1,74$ & $2,02-2,32$ \\
\hline \multicolumn{8}{|l|}{ Lockergesteine } \\
\hline Kies, trocken & $2,7-2,8$ & $0,4-0,5$ & 0,4 & $1,4-1,6$ & \multirow[b]{2}{*}{$1,93-2,30$} & \multirow[b]{2}{*}{$0,61-2,58^{*}$} & \multirow[b]{2}{*}{$1,21-2,21^{*}$} \\
\hline $\begin{array}{l}\text { Kies, wasserge- } \\
\text { sättigt }\end{array}$ & ca. 2,7 & 1,8 & 1,8 & ca. 2,4 & & & \\
\hline Sand, trocken & $2,6-2,7$ & $0,3-0,8$ & 0,4 & $1,3-1,6$ & \multirow[b]{2}{*}{$1,62-2,0$} & \multirow[b]{2}{*}{$0,33-2,46^{\star}$} & \multirow[b]{2}{*}{$1,46-2,49^{*}$} \\
\hline $\begin{array}{l}\text { Sand, wasserge- } \\
\text { sättigt }\end{array}$ & $2,6-2,7$ & $1,7-5,0$ & 2,4 & $2,2-2,9$ & & & \\
\hline $\begin{array}{l}\text { Ton/Schluff, } \\
\text { trocken }\end{array}$ & - & $0,4-1,0$ & 0,5 & $1,5-1,6$ & \multirow{2}{*}{1,70} & \multirow{2}{*}{$1,23-1,61^{*}$} & \multirow{2}{*}{$2,04-2,23^{\star}$} \\
\hline $\begin{array}{l}\text { Ton/Schluff, } \\
\text { wassergesättigt }\end{array}$ & - & $0,9-2,3$ & 1,7 & $1,6-3,4$ & & & \\
\hline
\end{tabular}




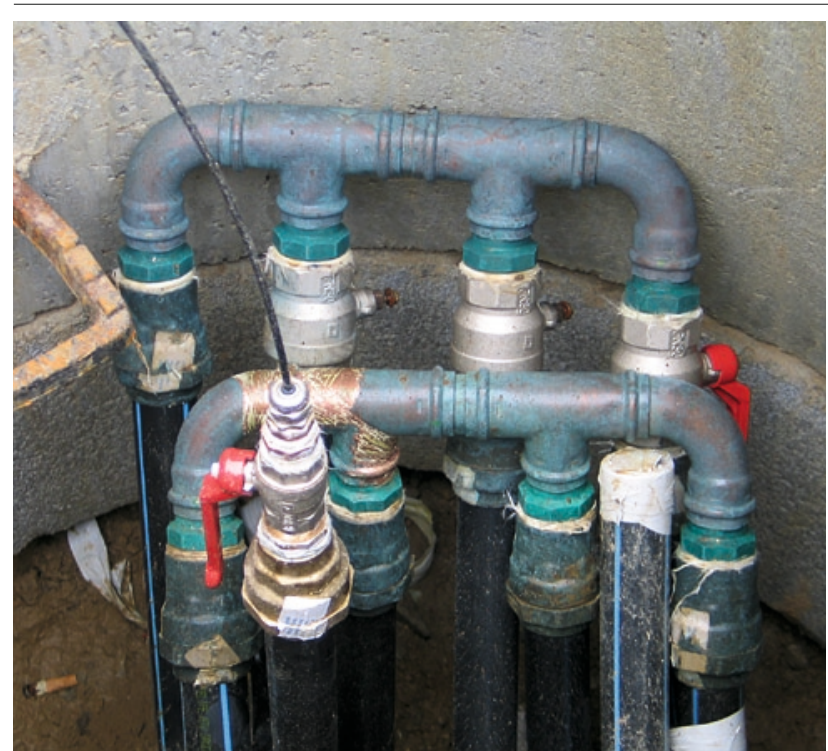

Abb. 4: Sondenkopf mit Glasfaserkabel.

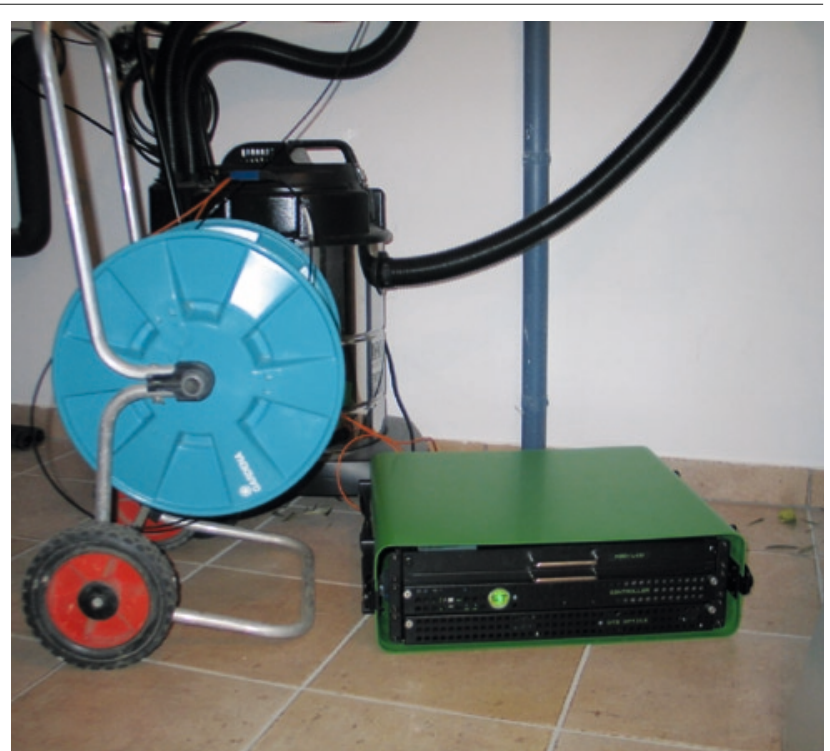

Abb. 5: Aufnahmegerät.
Die untersuchten Schotter zeigen ebenfalls eine große Variabilität. Der kleinste gemessene Wert liegt bei $\lambda=0,90 \mathrm{~W} / \mathrm{mK}$ (Hochterrassenschotter), der Maximalwert bei $\lambda=2,74 \mathrm{~W} / \mathrm{mK}$ (Niederterrassenschotter). Die beiden Schotter besitzen ähnliche Kornsummenkurven, die höhere Wärmeleitfähigkeit des Niederterrassenschotters ist jedoch auf die höhere Lagerungsdichte und den höheren Wassergehalt zurückzuführen. Im Vergleich liegen die gemessenen $\lambda$-Werte auch im bergfeuchten Zustand der Messproben bereits höher als der angegebene Literaturwert bei vollständiger Wassersättigung. Eine deutliche Abweichung zu den Literaturangaben ist hier erkennbar.

Die gemessenen Wärmekapazitäten der Sande und Schotter stimmen gut mit den Rechenwerten der Literatur überein.

Bei den untersuchten feinkörnigen Böden zeigen sich keine großen Unterschiede in den gemessenen thermischen Parametern. Die Messwerte der Wärmeleitfähigkeit schwanken zwischen 1,23 und 1,61 W/ $\mathrm{mK}$, die der Wärmekapazität zwischen 2,0 und $2,2 \mathrm{MJ} / \mathrm{m}^{3} \mathrm{~K}$. Im Vergleich entsprechen die gemessenen $\lambda$-Werte im bergfeuchten Zustand der Messproben in etwa dem angegebenen Literaturwert bei vollständiger Wassersättigung.

\subsection{Indirekte (qualitative) Bestimmung der thermischen Eigenschaften des Untergrundes}

Der Einfluss des Untergrundes auf die Entzugsleistung von Erdwärmesonden kann indirekt auch mittels der ortsauflösenden faseroptischen Temperaturmessung dar- gestellt werden. Ähnlich wie beim Thermal Response Test wird dabei der Temperaturverlauf beim Entzug bzw. bei Zufuhr von Wärmeenergie gemessen. Die Temperaturmessung erfolgt dabei jedoch nicht punktuell am Sondenkopf (Soleeintrittstemperatur und -austrittstemperatur), sondern über die gesamte Sondenlänge. Dabei wird ein Glasfaserkabel in den Vorlauf der Erdwärmesonde bis zur Endteufe eingebracht. Über ein T-Stück mit dichter Verschraubung wird das Kabel am Sondenkopf aus der Sonde heraus und zum Aufzeichnungsgerät geführt. Dadurch kann im Betrieb der Wärmepumpenanlage direkt die Soletemperatur in der Erdwärmesonde in Abständen von 0,5 Meter gemessen werden. Der Abstand der Messpunkte entlang des Kabels beträgt dabei 0,5 Meter

Dies ist ein wesentlicher Vorteil dieser Messmethode gegenüber anderen Temperaturmessmethoden (z. B. Messsonde Fisch).

In Abbildung 4 ist der Sondenkopf mit dem herausgeführtem Glasfaserkabel dargestellt, Abbildung 5 zeigt das Aufnahmegerät.

Die aufgezeichneten Temperaturprofile geben zusammen mit der gleichzeitig gemessenen Entzugsleistung Aufschluss über die thermischen Eigenschaften des Untergrundes im Nahbereich der Sonde. Von besonderem Interesse sind dabei die instationären Betriebszustände der Wärmequellenanlage (Einschalten, bzw. Ausschalten der Wärmepumpe).

Der Einsatz dieser Messmethode erfolgt erstmals in Österreich in einem Forschungsprojekt über die Energieeffizienz von Erdwärmesonden. Das Projekt wird aus Mitteln des Klima- und Energiefonds gefördert und im Rahmen des Programms „ENERGIE DER ZUKUNFT“ durchgeführt.

In Abbildung 6 sind Tiefenprofile der Soletemperatur einer 100 Meter langen Erdwärmesonde während des Betriebes (Wärmeentzug) dargestellt.

Die strichlierte horizontale Linie im Diagramm stellt dabei den Eintritt der Sole in die Erdwärmesonde dar. Das Tiefenprofil 1 wurde noch vor dem Einschalten der Wärmepumpe aufgezeichnet und kann als quasi stationärer Temperaturverlauf angesehen werden. Die weiteren Temperaturprofile 2 bis 6 zeigen die Entwicklung der Soletemperatur bei permanentem Wärmepumpenbetrieb im zeitlichen Abstand von 30 Minuten. Sehr deutlich ist die kontinuierliche Zunahme der Soletemperatur über die Sondenlänge ersichtlich. Abgesehen von geringen Temperaturschwankungen sind keine signifikanten Unstetigkeiten im Temperaturverlauf erkennbar. Das zugehörige Bohrprofil zeigt ab 10 Meter unter Gelände einen homogenen Untergrundaufbau. Diese Homogenität des Untergrundes spiegelt sich in der bereits beschriebenen stetigen Zunahme der Soletemperatur wieder.

Abbildung 7 zeigt ebenfalls Tiefenprofile der Soletemperatur einer 50 Meter langen Sonde. Deutlich erkennbar ist hier der sehr unstete Verlauf der gemessenen Temperaturen. Das Profil 1 stellt dabei wiederum einen quasi stationären Zustand vor Inbetriebnahme der Wärmepumpe dar. Die Profile 2 bis 4 zeigen den Verlauf der Soletemperatur in der Sonde im zeitlichen Abstand von 30 Minuten. 


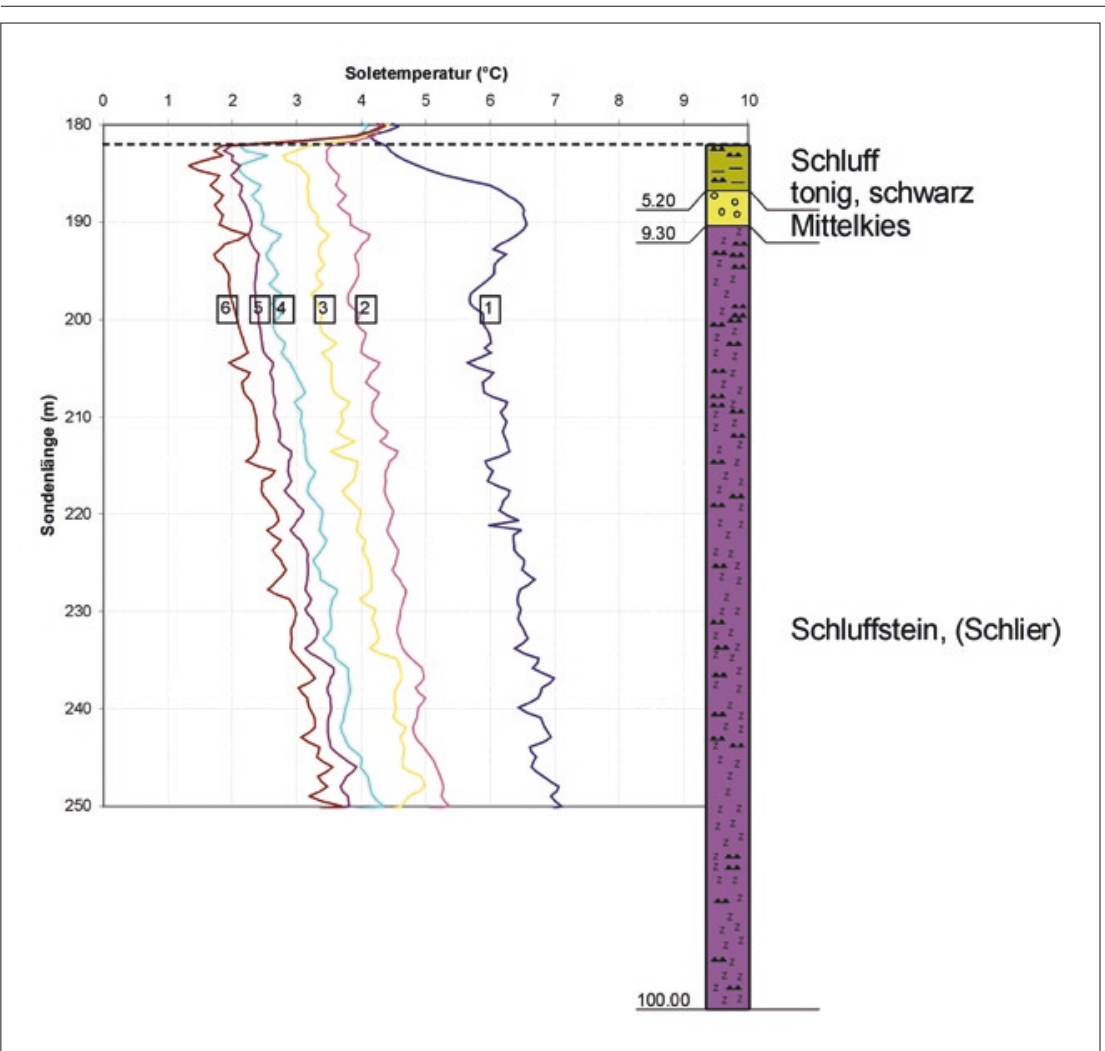

Abb. 6: Tiefenprofile, homogener Untergrund.

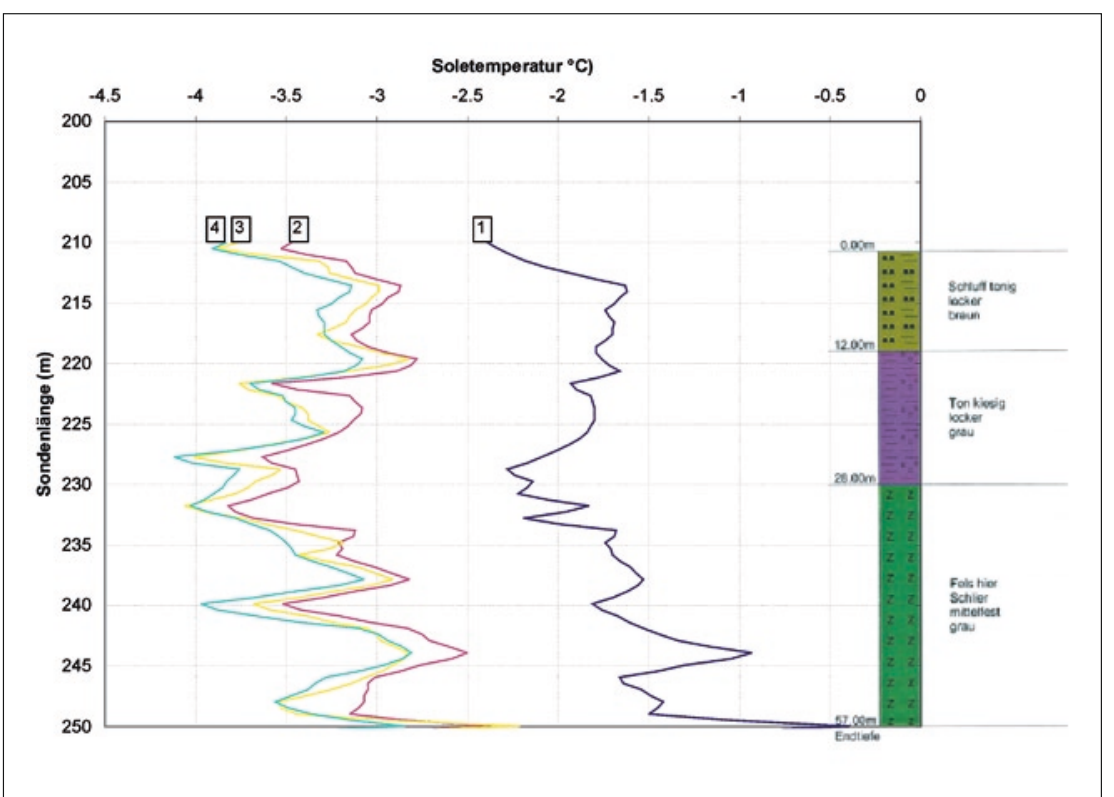

Abb. 7: Tiefenprofile, inhomogener Untergrund.

Das zugehörige Bohrprofil zeigt generell zwei unterschiedliche Schichtkomplexe. Vom Hangenden bis $28 \mathrm{~m}$ Tiefe handelt es sich um ein feinkörniges Lockergestein, im Liegenden um Festgestein. In den Temperaturprofilen ist der Schichtwechsel sehr deutlich erkennbar. Erst bei Erreichen des Festgesteins steigt die Soletemperatur an, darüber stagniert thermischen Untergrundverhältnissen durchaus abgeleitet werden.

Mit der Methodik der ortsauflösenden faseroptischen Temperaturmessung lässt sich auch der Einfluss des Grundwassers auf die Soletemperatur und somit auch auf die Entzugsleistung darstellen.

Fließendes Grundwasser bewirkt, je nach Fließgeschwindigkeit, einen Wärmetransport (Advektion von Wärme zur Erdwärmesonde hin), der durch die hohe spezifische Wärmekapazität des Wassers begünstigt wird. Dabei spielt die Gesamtwassermenge, die durch den Grundwasserleiter fließt, die entscheidende Rolle.

In Abbildung 8 sind Temperaturprofile in einer 100 Meter langen Erdwärmesonde, die während und nach eines Thermal Response Tests aufgezeichnet wurden, dargestellt.

Aufgrund der turbulenten Strömung der Sole und der geringen Grundwassermächtigkeit zeigt sich während des Testbetriebes keine nennenswerte Temperaturabsenkung im Bereich des strömenden Grundwassers. Erst nach Abschaltung der Heizung und der Umwälzpumpe wird eine deutliche Temperaturanomalie im Grundwasserbereich ersichtlich. Die Temperaturprofile zeigen hier einen raschen $\mathrm{Ab}$ transport der Wärme und somit ein starkes Absinken der Soletemperatur im Bereich von 8 bis 12 Meter unter Gelände.

\section{Einflussfaktor Verpressmaterial}

Bei der Herstellung von Erdwärmesonden müssen die eingesetzten Materialien eine lange Lebensdauer aufweisen, damit die Funktionsdauer der gesamten Anlage weit über der langen Amortisationsdauer liegt. Daher ist es notwendig bei der Herstellung und den dabei verwendeten Materialien hohe Qualitätsstandards anzuwenden. Eine Festlegung von nachvollziehbaren und auf der Baustelle überprüfbaren Qualitätskriterien und Anforderungen - auch für die verwendeten Verpressmaterialien- ist daher unumgänglich. In der Praxis haben sich zum Verfüllen der ausgebauten Bohrungen Fertigprodukte durchgesetzt. Zum Teil kommen jedoch auch Verfüllmaterialien zum Einsatz, welche an der Baustelle aus mehreren Komponenten angemischt werden. Diese basieren meist auf Erfahrungswerten der Brunnenbauer und werden unter stark variierenden Randbedingungen hergestellt und in das Bohrloch eingebracht. Überprüfbare Qualitätskriterien und einheitliche Bewertungsmöglichkeiten für an der Baustelle herge- 


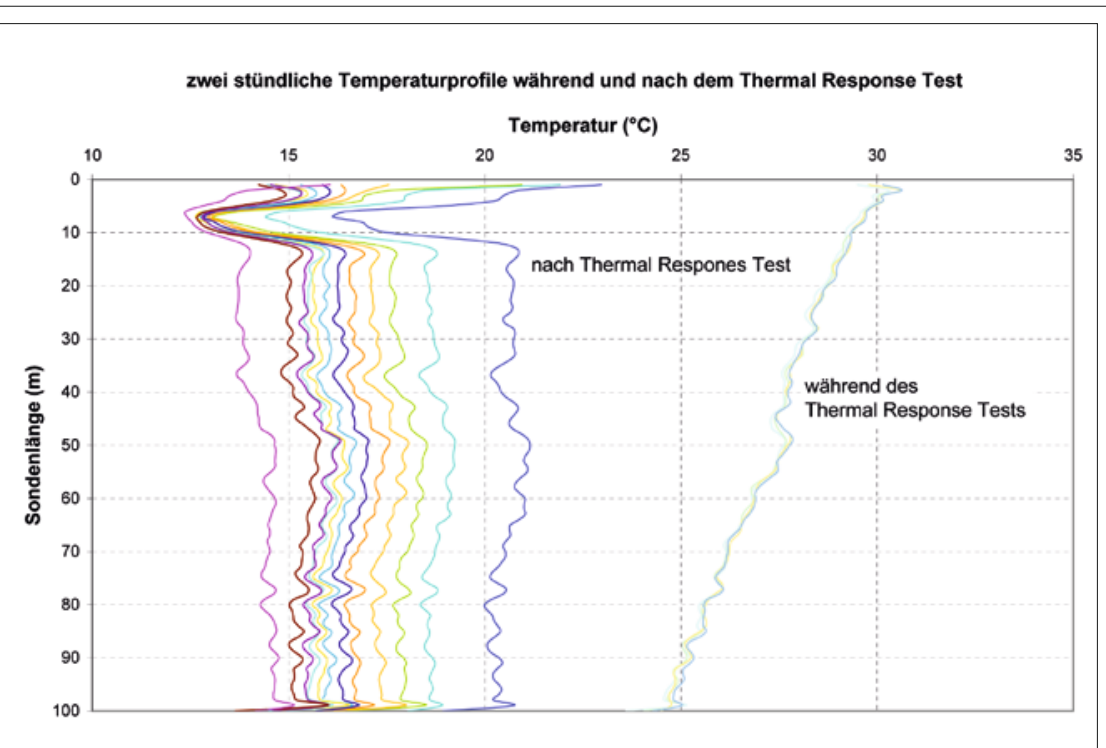

Abb. 8: Temperaturprofile während eines Thermal Response Tests.

stellte Suspensionen aus Fertigprodukten als auch auf Basis von Rezepturen hergestellte Mischungen bestehen meist nicht.

Aus diesem Grund führten die Austrian Institute of Technology GmbH - AIT gemeinsam mit der Oberösterreichische Boden- und Baustoffprüfstelle GmbH im Auftrag der Oberösterreichischen Landesregierung Eignungsuntersuchungen an Verpressmaterialien durch. Die dabei untersuchten Materialien setzten sich aus sieben Fertigprodukten und drei Baustellenmischungen auf Basis von bewährten Rezepturen zusammen.

\subsection{Generelle Anforderungen an Verpressmaterialien}

Im Hinblick auf eine optimale Energieausbeute sowie die bei Behörden im Vordergrund stehenden wasserwirtschaftlichen Aspekte von Erdwärmesonden können folgende Anforderungen definiert werden:

- Wirksame Abdichtung von Grundwasserstockwerken gegeneinander zur Verhinderung von hydraulischen Kurzschlüssen. Nach dem vollständigen Abbinden der Verpresssuspension sollen sich die natürlichen Druck- und Fließverhältnisse in den durchörterten Aquiferen wieder einstellen.

- Minimierung der thermischen Eintrittsverluste in die Erdwärmesonde durch Realisierung eines niedrigen Bohrlochwiderstandes.

- Langzeitbeständige effektive Ankopplung der Sondenverrohrung an den umgebenden Untergrund.

- Nachgewiesene Umweltverträglichkeit
- Trichterauslaufzeit, TAZ

- Suspensionsdichte, $\rho_{\mathrm{s}}$

- Fließgrenze, kurz $\tau_{\mathrm{f}}$

- Filtratwasserabgabe

- Absetzmaß

Anhand der Parameter Fließgrenze, Filtratwasserabgabe und Absetzmaß soll der Einfluss der Suspensionseigenschaften auf das Abdichtungsverhalten sowie die thermischen Eigenschaften erläutert werden.

Die an den untersuchten Mischungen ermittelten Fließgrenzen sind in Abbildung 9 dargestellt. Die Werte variieren dabei von $\tau_{\mathrm{f}}=16 \mathrm{~N} / \mathrm{mm}^{2}$ bis $\tau_{\mathrm{f}}=66 \mathrm{~N} / \mathrm{mm}$. Die Fließfähigkeit der Suspension wird im Wesentlichen von der Fließgrenze $\tau_{\mathrm{f}}$ beeinflusst. Steigt die Schubspannung während des Fließvorganges in der Suspension über den Wert der Fließgrenze $\tau_{\mathrm{f}}$, endet der Fließvorgang und die Suspension kommt zum Stehen. Das bedeutet die Fließfähigkeit einer Suspension ist umso größer, je kleiner ihre Fließgrenze ist. Eine fließfähige Konsistenz ist jedoch Voraussetzung für eine hohlraumfreie und abdichtende Verfüllung.

Die Fließfähigkeit wird im Wesentlichen durch den W/F-Wert sowie durch das Vorhandensein von quellfähigen Phasen in der Suspension beeinflusst. Hohe W/F-Werte führen allgemein zu geringen Fließgrenzen und somit zu fließfähigen Suspensionen. Die sich dabei einstellenden Suspensionsdichten sind jedoch meist zu gering. Sind in der Suspension Quelltone vorhanden binden diese überschüssiges Wasser, was auch bei hohen W/F- Werten zu hohen Fließgrenzen führen kann.

Die im Filterabpressversuch ermittelten Filtratwasserabgaben sind in Abbildung 10 dargestellt. Generell zeigt eine große Filtratwasserabgabe, dass freies, ungebundenes Wasser in der Suspension vorhanden ist, welches nicht von hydraulischen Bindemitteln oder quellfähigen Phasen aufgenommen werden kann. Dieses freie Wasser kann im Bohrloch in durchlässiges Gebirge abfiltrieren, was zu einem Volumenverlust in der Suspension führt. Eine große Filtratwasserabgabe deutet bei den Fertigprodukten auf einen geringen Anteil, bzw. das Fehlen von quellfähigen Phasen hin, da diese einen Großteil des freien Wassers anlagern und dieses im Filterabpressversuch nur zu einem geringen Teil abgeben.

Bleibt das freie Wasser in der Suspension so führt es nach dem Abbinden zu einer höheren Porosität. Dies erhöht die Was- 


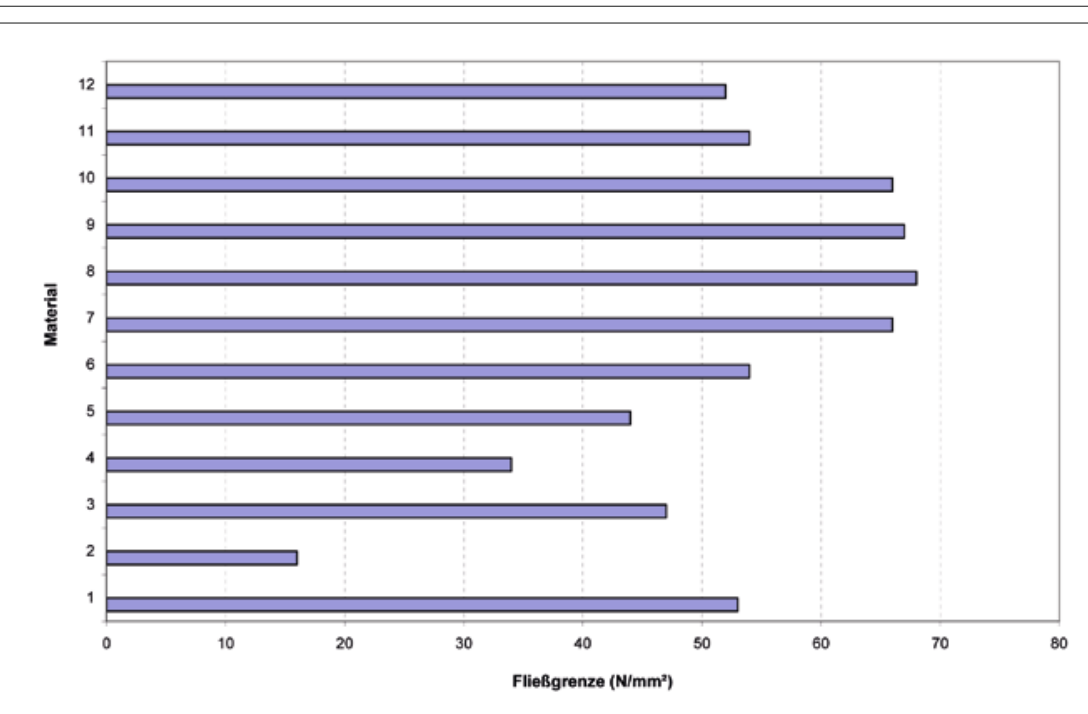

Abb. 9: Ermittelte Fließgrenzen der Suspensionen.

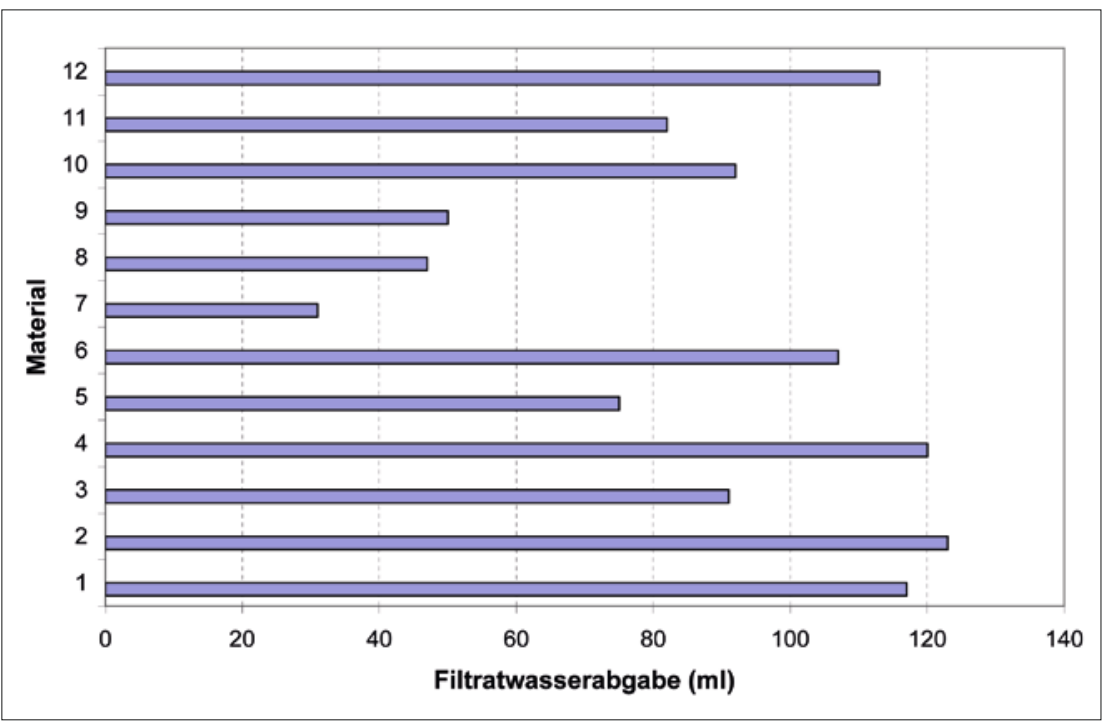

Abb. 10: Ermittelte Filtratwasserabgaben.

serdurchlässigkeit, da mehr Porenraum für die Wasserbewegung zur Verfügung steht. Kann das Porenwasser aus der erhärteten Suspension austreten und Luft in den Porenraum eindringen, so kommt es zu einer starken Verringerung der Wärmeleitfähigkeit, da Luft eine etwa 100-mal geringere Wärmeleitfähigkeit als Wasser besitzt.

Das nach 3 Stunden gemessene Absetzvolumen zeigt unter den gemessenen Suspensionsparametern den größten Streuungsbereich auf $(0,9$ bis max. 33,1 ml). Ähnlich dem Bluten von Beton kommt es durch Sedimentationseffekte zum Absetzen von klarem Wasser an der Oberfläche. Ein hohes Absetzvolumen deutet auf instabile Suspensionen hin. Als Folge stellt sich ein Schwinden des Materials ein. gleichzeitig in das Anmachwasser eingerührt, so ergeben sich deutlich schlechtere Suspensionseigenschaften.

\subsection{Abbindeverhalten und Wasser- durchlässigkeit}

Das zeitliche Abbindeverhalten von Verpressmaterialien kann im Labor durch einaxiale Druckversuche an Probekörpern bei unterschiedlichen Abbindezeitpunkten $(7,14,28$ und 56 Tagen) ermittelt werden. Die so gemessenen Festigkeiten der untersuchten Materialien sind in Abbildung 11 dargestellt.

Neben der Endfestigkeit ist dabei auch der Verlauf des Festigkeitszuwachses interessant. Einige Materialien zeigen einen proportionalen Zuwachs über die Abbindezeit. Bei anderen ist der Festigkeitszuwachs zu Beginn gering und erst nach 14 Tagen kommt es zu einem überproportionalen Anstieg. Diese Verzögerung in der Hydratation des Zementes dürfte aufgrund von speziellen Zusatzmitteln oder durch Quelltone verursacht worden sein.

Für Schlitzwandmassen wird eine Mindestdruckfestigkeit nach 28 Tagen von $\mathrm{q}_{\mathrm{f}}=0,3 \mathrm{~N} / \mathrm{mm}^{2}$ gefordert. Dies ist im Hinblick auf eine ausreichende Erosionsstabilität wichtig. Zwei Rezepturen erreichen diese Vorgabe bei weitem nicht. Bei diesen Baustellenmischungen scheint einerseits der W/F-Wert zu hoch, andererseits das Verhältnis von Zement zu Quellton zu gering zu sein.

In Hinblick auf eine rasche Abdichtungswirkung soll bereits nach 24 Stunden eine möglichst hohe Anfangsfestigkeit gegeben sein.

Die Ermittlung des Wasserdurchlässigkeitskoeffizienten erfolgte an 28 Tage alten Probekörpern. Die gemessenen Werte schwanken zwischen $\mathrm{k}_{\mathrm{f}}=1 \mathrm{E}-9$ und $1 \mathrm{E}-8 \mathrm{~m} / \mathrm{s}$, siehe Abbildung 12. Bei Lockergesteinsböden entspricht dies einem schwach bis sehr schwach durchlässigen Boden wie z.B. Schluff-Tongemischen („Grundwasserstauer").

Bei den Baustellenmischungen führen hohe W/F Wert in Kombination mit dem geringen Verhältnis von Zement zu Quellton zu durchlässigeren Probekörpern. Die während der Hydratation des Zementes entstehenden kristallinen Phasen verschließen nur Teile des Porenraumes, sodass noch genügend offene Kanäle als Wasserwegigkeiten offen bleiben.

Von der EAU (1990) wird ein Durchlässigkeitsbeiwert von $\mathrm{k}_{\mathrm{f}}<5$ E-8 m/s für konventionelle Dichtmassen zur Bautechni- 


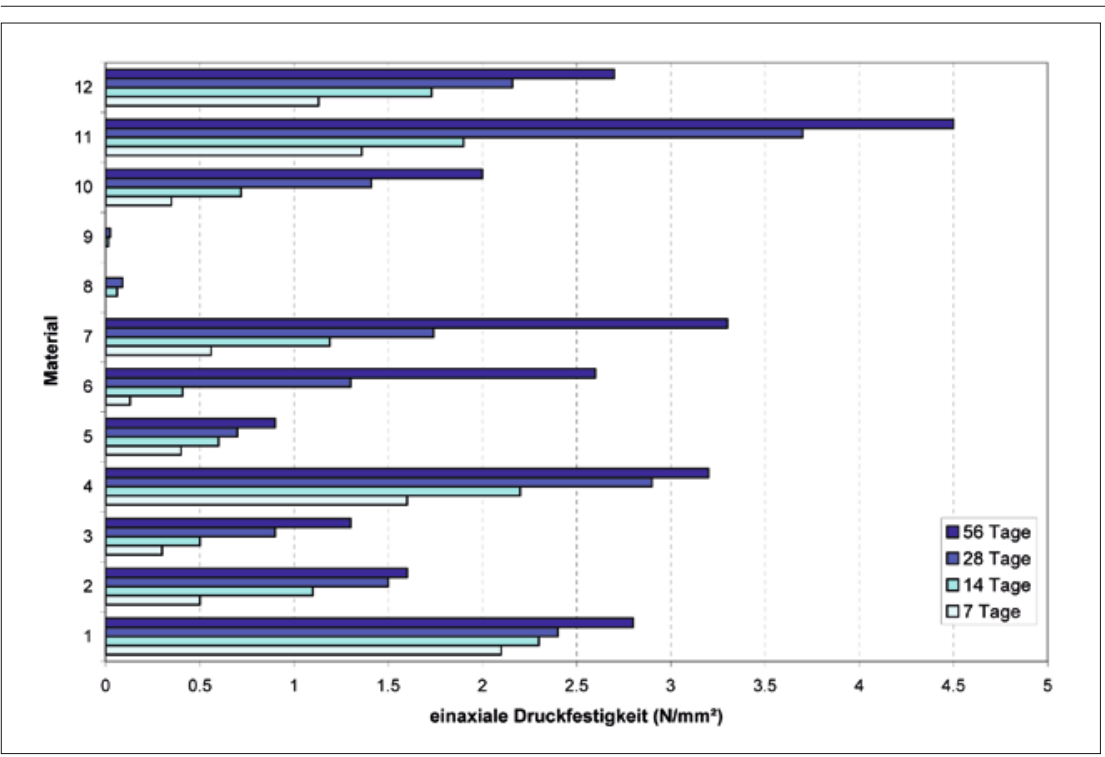

Abb. 11: einaxiale Druckfestigkeiten zu versch. Abbindezeitpunkten.

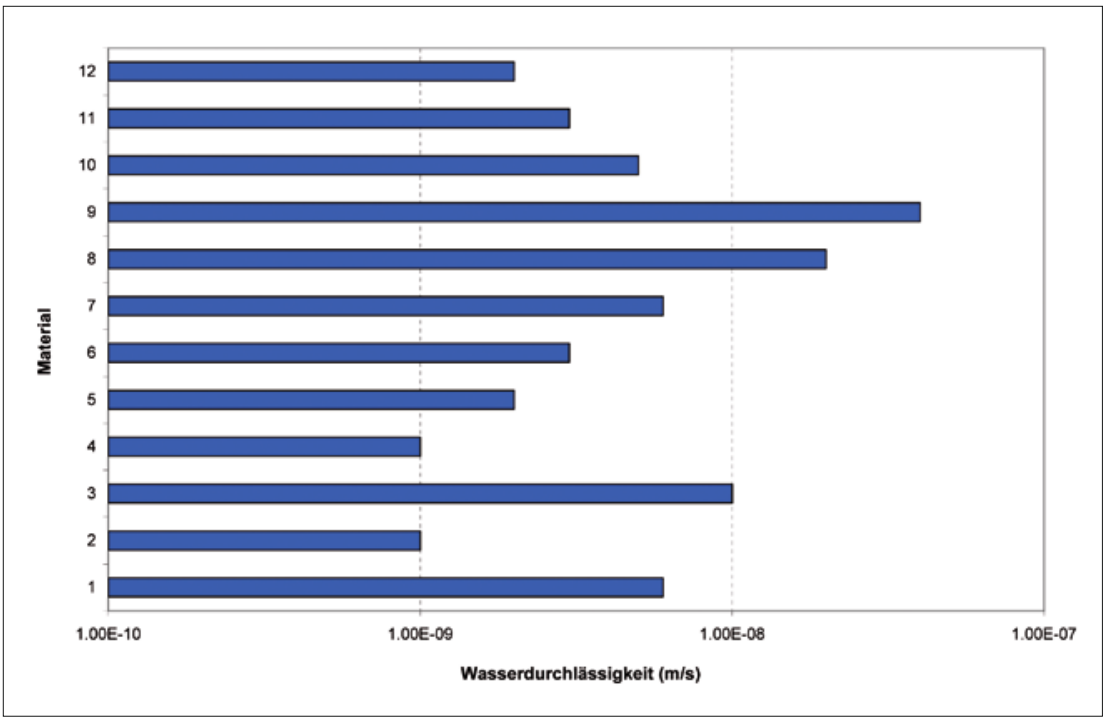

Abb. 12: Wasserdurchlässigkeiten nach 28 Tagen.

schen Abdichtung gegen Grundwasser gefordert. Dabei ist jedoch unklar ob es sich um die reine Materialdurchlässigkeit oder um die Systemdurchlässigkeit samt allen Einbauten handelt.

Die im Labor ermittelten Durchlässigkeiten geben nur die reine Materialdurchlässigkeit der abgebundenen Suspensionen wider. Die Systemdurchlässigkeit, d. h. die Durchlässigkeit der Verpressung samt Sondenverrohrung ist um ca. um eine halbe bis eine Zehnerpotenz höher anzusetzen.

\subsection{Frostverhalten der Verpress- materialien}

Bei Verpressmaterialien handelt es sich, wie bei Beton, um hydraulisch gebundene
Der Zusammenhang zwischen Porenradius und Gefrierpunkt macht deutlich, dass ein Teil des Porenwassers auch bei Temperaturen weit unter dem Gefrierpunkt ungefroren bleibt.

In Folge kommt es zu einer Gefügezerstörung und zur Bildung von Mikrorissen. Weiters kann es zur so genannte „Mikroeislinsenbildung" kommen. Der erforderliche Druck zum Transport des Wassers wird von entstehenden Eislinsen, die zuerst in den größeren Kapillarporen während der Gefrierphase entstehen, verursacht. Mit zunehmender Unterkühlung wachsen die Mikroeislinsen in kleinere Poren hinein und drücken ungefrorenes Wasser aus den Gelporen zum makroskopischen Eis hinaus (Gefrierschwinden) und friert dort an.

Dieser Vorgang beginnt an der Oberfläche und setzt sich nach innen fort. Die dann in der Auftauphase eintretende zusätzliche Wasseraufnahme (z.B. extern aus der Prüflösung) wird durch die zunehmend einsetzende Entspannung des Mikrogefüges durch den Abbau der Druckdifferenz zwischen ungefrorenem Wasser und Eis ausgelöst und führt zu einer künstlichen Wassersättigung.

Bei der Wirkung des Forstes auf das Verpressmaterial spielen die Zeit und der Temperaturverlauf während der FrostTau-Periode eine bedeutende Rolle. Ein langsamer Gefriervorgang fördert die Eislinsenbildung, setzt jedoch die Sprengwirkung des sich in den Hohlräumen bildenden Eises in Folge der Ausweichbewegung des Wassers stark herab. Ein schneller Gefriervorgang fördert die Sprengwirkung des Wassers, vermindert jedoch die Bildung von Eislinsen.

Die Auswertung der visuellen Beurteilung an den Probekörper nach der FrostTauwechselbeanspruchung zeigte fast durchgehend leichte bis schwere Schäden in Form von Rissen und Abplatzungen bis hin zum Bruch des Probekörpers. Die einzige Ausnahme bilden die Probekörper eines Fertigproduktes. Hier waren keine sichtbaren Schäden erkennbar. Exemplarisch sind in Abbildung 14 und 15 zwei Probekörper mit Rissen in der Stirnfläche sowie ein durch die Frosteinwirkung gebrochener Probekörper dargestellt.

Als eine messbare Beurteilung des Einflusses von Frost-Tauwechseln auf das Verpressmaterial erfolgte die Ermittlung der einaxialen Druckfestigkeit an befrosteten zylindrischen Probekörpern sowie an gleichaltrigen Probekörpern welche bei Raumtemperatur gelagert wurden.

Aus dem Vergleich beider Werte lassen sich Rückschlüsse auf strukturelle Schäden 


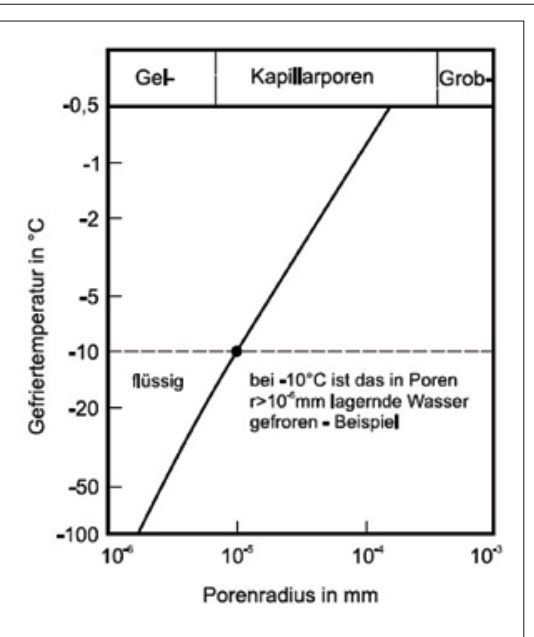

Abb. 13: Gefriertemperatur vom Wasser in Abhängigkeit der Porengröße,

Quelle: Budelmann, H., Baustoffkunde 2.

der befrosteten Proben ziehen. In Abbildung 16 sind die Ergebnisse dieser Versuche zusammengestellt. Die Versuchsreihe „Probe ohne Befrostung" beinhaltet dabei die einaxialen Druckfestigkeiten der gleichaltrigen Parallelproben, die Reihe „Probe mit Befrostung “ die Druckfestigkeiten der befrosteten Proben. Die Prozentangaben geben den Abfall der Druckfestigkeit bezogen auf die unbefrostete Probe wider.

Der Abfall an Druckfestigkeit beträgt wenige Prozentpunkte bis hin zu $49 \%$. Diese Ergebnisse zeigen deutlich, dass es zu einer Gefügeveränderung bzw. Gefügezerstörung des abgebundenen Verpressmaterials kommt.

Im Zuge der Befrostungsversuche wurden ebenfalls die Wärmeleitfähigkeiten von befrosteten und unbefrosteten Probekörpern gemessen.

Die ermittelten Wärmeleitfähigkeiten bewegen sich bei den Fertigprodukten zwischen 2,39 W/mK und 0,77 W/mK. Die von den Herstellern oft genannte Wärmeleitfähigkeit von $2 \mathrm{~W} / \mathrm{mK}$ wird daher nur von einem Produkt erreicht. Die gemessenen Baustellenmischungen besitzen aufgrund der unterschiedlichen Wassergehalte beim Anmischen auch unterschiedliche Wärmeleitfähigkeiten. Die gemessenen Werte liegen zwischen $0,72 \mathrm{~W} / \mathrm{mK}$ und $0,86 \mathrm{~W} / \mathrm{mK}$.

Der Vergleich zwischen den Wärmeleitfähigkeiten von Probekörpern mit und ohne Frosttauwechselbeanspruchung, wie in Abbildung 17 dargestellt, zeigt an befrosteten Probekörper durchwegs geringere Wärmeleitfähigkeiten. Dies kann wie bei der einaxialen Druckfestigkeit auf eine Veränderung der Gefügestruktur durch Mikrorisse hervorgerufen werden, was zu einer Verschlechterung der Wärmeleitung im Material führt. In den Diagrammen ist weiters der Rückgang der Wärmeleitfähigkeit als Prozentwert zu jedem Material eingetragen.

\section{ZUSAMMENFASSUNG}

Die Entzugsleistung und damit die Länge der Erdwärmesonde sind von den geothermischen Eigenschaften des anstehenden Bodens am jeweiligen Standort abhängig. Die dabei wesentlichen Parameter sind die Wärmeleitfähigkeit $\lambda$ und die Wärmekapazität c. Vergleichsmessungen dieser Parameter in Situ ergeben dabei im
Vergleich zu einschlägigen Literaturwerten ähnliche Ergebnisse.

Bei den Festgesteinen lassen sich Abweichungen auf Schwächungen (Klüfte, Mikrorisse) und Verwitterung der untersuchten Gesteine zurückführen. Es ist daher durchaus davon auszugehen, dass im massigen ungestörten Gesteinsverband höhere Wärmeleitfähigkeiten als die in den oberflächennahen Aufschlüssen gemessenen auftreten.

Bei den Lockergesteinen sind die Körnungslinie sowie die Lagerungsdichte und Wassergehalt maßgebend für die Wärmeleitfähigkeit. Unterschiede zu den Literaturangaben zeigen sich besonders bei Schottern. Diese weisen im bergfeuchten Zustand höhere $\lambda$-Werte auf als in der Literatur angegeben.

Weiters kann mit Hilfe der ortsauflösenden faseroptischen Temperaturmessung der Einfluss der Geologie und Hydrogeologie auf die sich im Betrieb der Erdwärmesonde einstellende Soletemperatur dargestellt werden. Ein homogener geologischer Untergrundaufbau spiegelt sich in einem stetig ansteigenden Temperaturprofil der Sole über die gesamte Sondenlänge wieder. Bei einem inhomogenen Untergrundaufbau zeigt auch der Verlauf der Soletemperatur Unstetigkeiten. Eine Differenzierung in unterschiedliche Abschnitte ist möglich. An einem Auswertemodell mit welchem eine tiefenbezogene Wärmeleitfähigkeit ermittelt werden kann, wird zurzeit gearbeitet.

Auch der Einfluss von Grundwasser kann mit dieser Methode dargestellt werden. Besonders nach dem Abschalten der
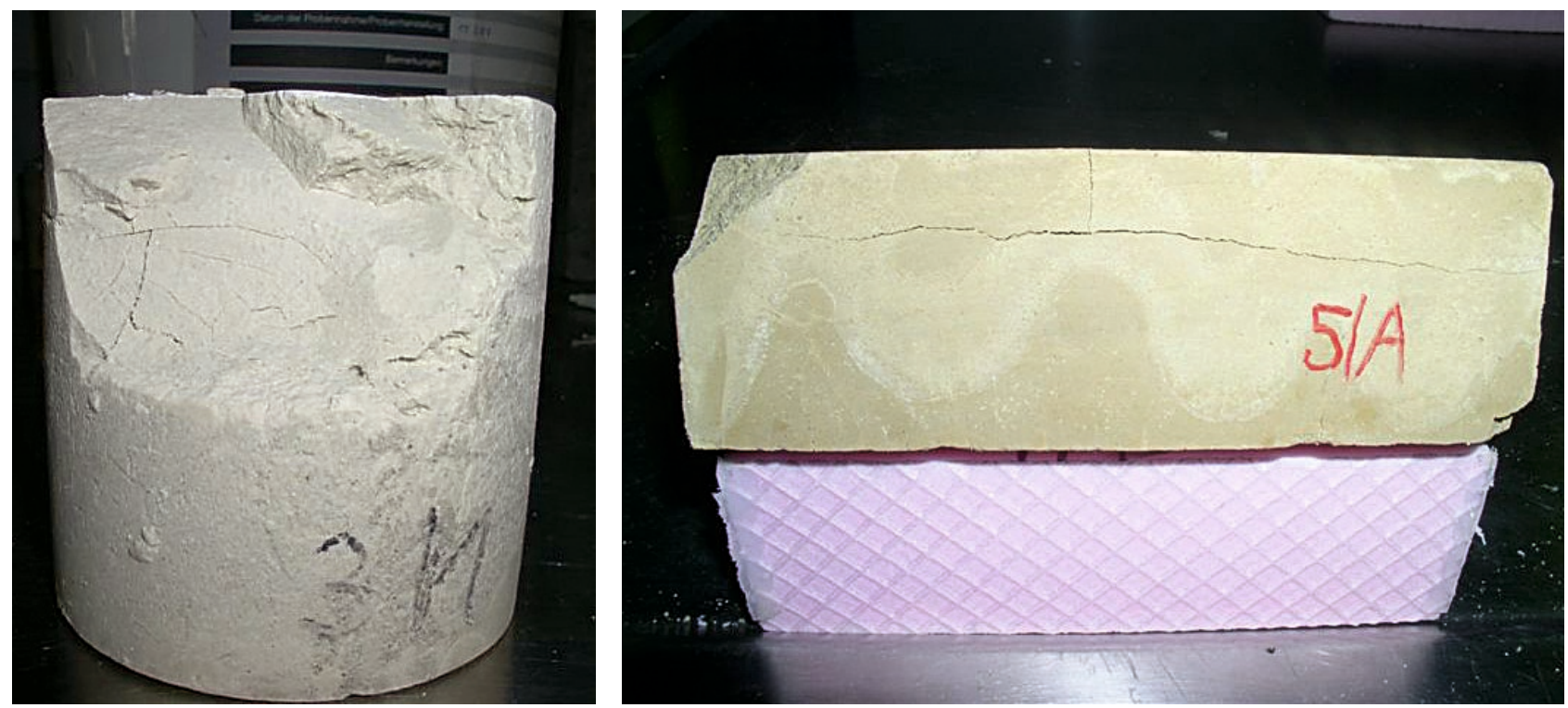

Abb. 14 (links) und 15 (rechts): gebrochene Probekörper nach 28 Frost-Tauwechselzyklen. 


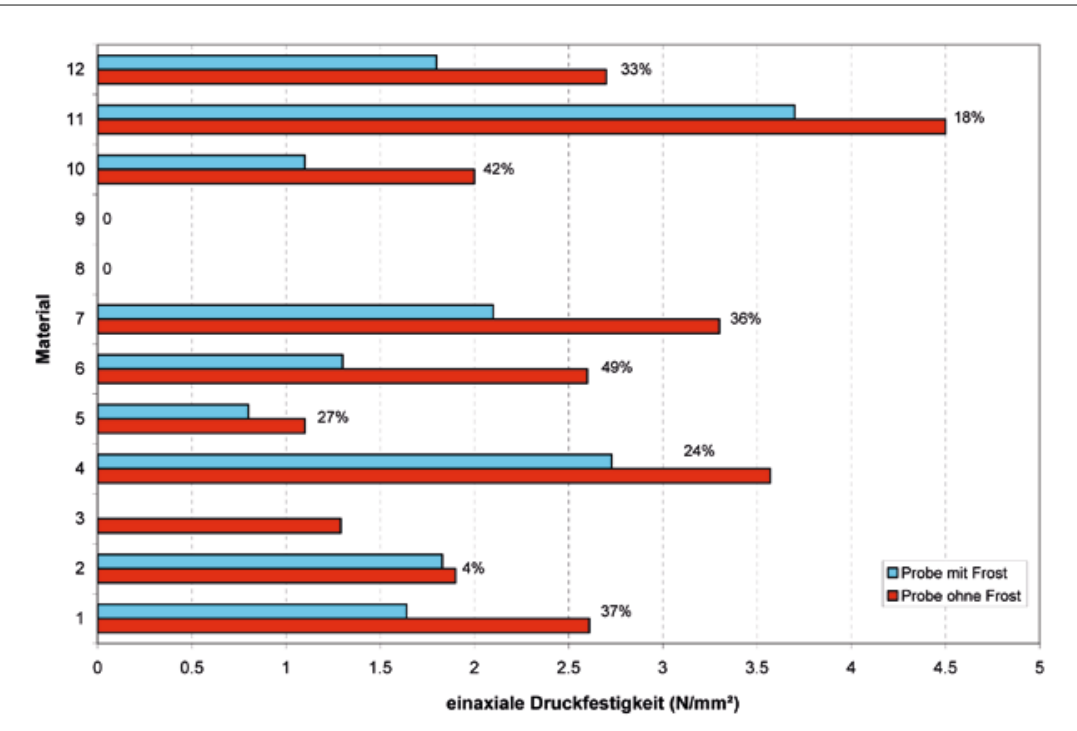

Abb. 16: einaxiale Druckfestigkeiten mit und ohne Befrostung.

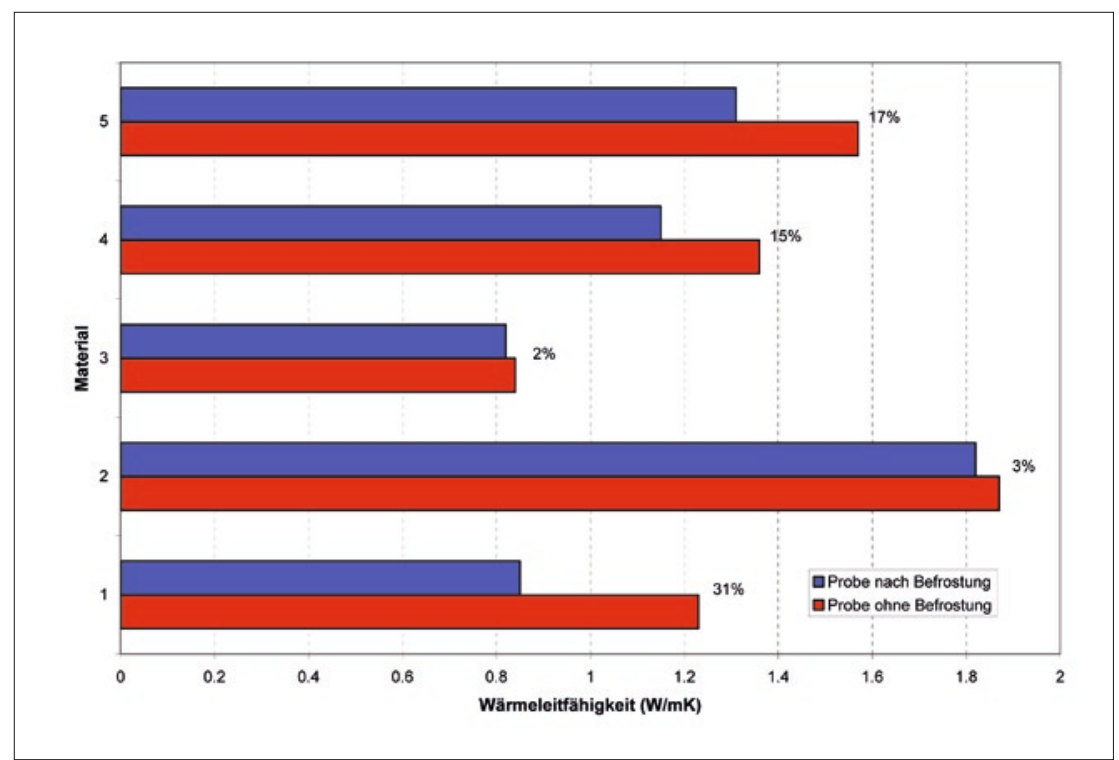

Abb. 17: Wärmeleitfähigkeit mit und ohne Befrostung.

Wärmepumpe zeigt sich in den periodisch gemessenen Temperaturprofilen die Stärke des Grundwassereinflusses durch eine mehr oder weniger ausgeprägte Unstetigkeit des Temperaturverlaufes, siehe Abbildung 8. An einer quantitativen Bewertung dieses Einflusses wird zurzeit ebenfalls gearbeitet.

Aus der Untersuchung von sieben am Markt angebotenen Fertigprodukten sowie von drei Rezepturen (Baustellenmischungen) zur Herstellung von Verpressmaterialien für Erdwärmesonden können in Hinblick auf die thermischen Eigenschaften und die wasserwirtschaftlich relevanten Aspekte folgende maßgebenden Anforderungen festgehalten werden.

\subsection{Nachhaltige und dauerhafte hydraulische Abdichtung}

Auf die Gewährleistung einer nachhaltigen und dauerhaften hydraulischen $\mathrm{Ab}$ dichtung haben bereits der Anmischvorgang sowie die damit einhergehenden Suspensionsparameter großen Einfluss. Besondere Bedeutung kommt dabei den Parametern Fließgrenze, Filtratwasserabgabe und Absetzmaß zu. Niedrige Fließgrenzen gewährleisten zwar ein leichteres Fließen und ein besseres Eindringen in den im Untergrund, bedingen jedoch meist einen höheren Anteil an freiem Wasser. Dieses freie Wasser führt im Zuge des Abbindevorganges zur Bildung von porösen Gefügestrukturen, was zur Erhöhung

der Wasserdurchlässigkeit führt. Die Parameter Filtratwasserabgabe und Absetzmaß geben Auskunft über das in der Suspension freie, nicht gebundene Wasser.

Ein rasches Abbinden nach dem Verpressen verhindert die Verschleppung des Verpressmaterials im Grundwasserleiter. Gleichzeitig sollen dabei Druckfestigkeiten des Materials erreicht werden, die eine Erosion des noch nicht vollständig abgebundenen Materials verhindern. Dadurch bleibt die abdichtende Funktion bis zum Erreichen der Endfestigkeit gewährleistet. Eine entsprechende Anfangsfestigkeit bedingt auch eine entsprechend geringe Wasserdurchlässigkeit. In den Modellversuchen konnte gezeigt werden, dass der Durchlässigkeitsbeiwert anfänglich höher ist als im ausgehärteten $\mathrm{Zu}$ stand (28 Tage). Mit zunehmender Abbindedauer nimmt die Durchlässigkeit jedoch ab. Weiters gilt es zu beachten, dass die Systemdurchlässigkeit der verpressten Sonde um ca. eine halbe bis eine Zehnerpotenz höher ist als die des reinen Verpressmaterials.

Auf die Dauerhaftigkeit der hydraulischen Abdichtung wirken sich oftmalige Frost-Tauwechsel negativ aus. Wie aus den Ergebnissen der Frost-Tauwechselversuche deutlich erkennbar ist, zeigen die Frost-Tauwechsel- beaufschlagten Proben deutlich geringere Festigkeiten als unbefrostete Proben, siehe Abbildung 16. Die strukturelle Schädigung der Probe zeigt deutliche Auswirkungen auf die Festigkeit. Die mit der Schädigung einhergehenden Rissbildungen und $\mathrm{Ab}$ lösungen von der Sondenverrohrung führen zu einer Erhöhung der Wasserdurchlässigkeit.

\subsection{Optimale Wärmeleitfähigkeit bei geringem thermischen Bohrloch- widerstand}

Wie bei der hydraulischen Abdichtungsfunktion ist auch für einen optimalen Wärmeübergang von anstehendem Untergrund in das Verpressmaterial eine vollständige Verfüllung aller Hohlräume und Klüfte erforderlich. Dazu ist eine gute Fließfähigkeit der Suspension notwendig. Weiters soll der Gehalt an freiem Wasser in der Suspension niedrig gehalten werden, da dieses zur Bildung von porösen Gefügestrukturen führt. Kann das Wasser aus den Poren migrieren und Luft eintreten, sinkt die Wärmeleitfähigkeit drastisch. Auch hohe Filtratwasserabgaben und ein hohes Absetzmaß können den Wärmetransport beeinträchtigen, da es zum 
Schwinden und in Folge zu Hohlraumbildungen kommen kann.

Der große Nachteil von Baustellenmischungen ist die im Vergleich zu den Fertigprodukten geringe Wärmeleitfähigkeit von $\lambda<1 \mathrm{~W} / \mathrm{mK}$. Dadurch ergibt sich ein vergleichsweise hoher Bohrlochwiderstand, der einen Energieverlust beim Eintritt in die Sonde darstellt. Eine leichte Erhöhung der Wärmeleitfähigkeit kann durch Zugabe von feinem Quarzsand erreicht werden, jedoch ist darauf zu achten, dass sich die Suspensionseigenschaften nicht verschlechtern (u.U. Sedimentationserscheinungen des Quarzsandes).

Ein hoher Bindemittelanteil in Verbindung mit einem geringem Wasser/Feststoff-Verhältnis erhöht ebenfalls die Wärmeleitfähigkeit. Durch den geringeren Anteil an freiem Wasser sinkt der Porenanteil und der Zementleim füllt den Porenraum vollständig aus.

Auch das Abbindeverhalten wird durch höhere Bindemittelanteile verändert, die
Anfangs- als auch die Enddruckfestigkeiten sind höher.

Wie in den Untersuchungen gezeigt werden konnte, beeinflusst die Gefügeschädigung aufgrund der Frost-Tauwechselbeanspruchung auch die Wärmeleitung im Verpressmaterial. Durch Mikrorisse und Ablösungen in den Übergangsbereichen wird der Wärmefluss gestört und die Wärmeleitfähigkeit des Materials sinkt, siehe Abbildung 16.

Geringe Wärmeleitfähigkeiten begünstigen in Verbindung mit tiefen Soletemperaturen das Einfrieren des Verpressmaterials, da nicht genügend Wärme aus dem Untergrund durch das Verpressmaterial zum Sondenrohr nachgeliefert werden kann. Die Schädigung durch oftmaligen Frost-Tauwechsel verschlechtert neben der Abdichtungsfunktion auch die Wirkeffizienz der gesamten Anlage. Es sollte daher auf jeden Fall thermisch verbessertes Verfüllmaterial verwendet werden. Auch ist bei der Anlagendimensionierung dar- auf zu achten, dass Soletemperaturen unter Null Grad nicht über einen langen Zeitraum auftreten.

Korrespondenz:

DI Richard Niederbrucker

Austrian Institute of Technology GmbH

Donau-City-Straße

1220 Wien

E-Mail: richard.niederbrucker@ait.ac.at

\section{LITERATUR}

Budelmann H (2006) Baustoffkunde 2 Geologische Bundesanstalt (2006) Geologie der Österreichischen Bundesländer. Oberösterreich. Geologische Karte 1:200.000.-Wien

Griesser JC, Rybach L. Temperatur- und Wär meleitfähigkeitsmessungen für Wärmeflussbestimmungen, geophysikalischer Feldkurs Hartge KH, Horn R (1999) Einführung in die Bodenphysik, 3. Auflage

Niederbrucker R, Steinbacher N (2007) Eignungsuntersuchung von Verpressmaterialien fü Erdwärmesonden, Technischer Endbericht VDI Regelblatt 4640 (2004) Thermische Nutzung des Untergrundes

SIA 384/6 (2009) Erdwärmesonden 